\title{
Numerical simulations of the shockwave induced collapse of bubbles near surfaces
}

\author{
${ }^{1}$ Nikolaos Bempedelis*; ${ }^{1}$ Yiannis Ventikos \\ ${ }^{I}$ Department of Mechanical Engineering, University College London, WC1E 7JE, United Kingdom
}

\begin{abstract}
In the study of collapsing bubbles and their relation to surface erosion, two mechanisms are identified as of major significance: the high-speed liquid jet and the water-hammer shock wave that is subsequently formed. In the current work, we suggest that secondary mechanisms such as the emission of shockwaves following the collapse of the bubble remains and the late-time wave interactions may also be of importance when it comes to surface erosion. We examine our hypothesis by considering the collapse of a bubble by a shock wave that runs in a direction that is parallel to a solid surface.
\end{abstract}

Keywords: cavitation; shock waves; bubble collapse; front tracking;

\section{Introduction}

Cavitation is a phenomenon that occurs in various applications and domains such as marine engineering, energy production and clinical medicine. The structural damage caused by bubbles collapsing near a surface has been a major drive in studies of cavitation. Two mechanisms have been suggested as primarily responsible for erosion: (i) the formation of a high-speed liquid jet and (ii) the water-hammer shock wave that is generated upon its impact on the bubble walls.

In the majority of applications, bubbles are present in clouds. Collapse dynamics are therefore dominated by interbubble effects: the presence of nearby bubbles disturbs the pressure field, whereas a bubble may collapse due to it being impacted by waves emitted from a neighboring bubble.

The shock-induced collapse of bubbles near surfaces has been mainly considered in the context of shockwave lithotripsy, where waves run in a direction that is normal to the surface [1,2]. The potential for damage in such configurations is great, as the main jet is directed towards the surface and the impact occurs at the side of the bubble that is closer to the wall. The emitted shockwave originates close to the surface and induces large pressures on it before decaying in strength. However, it has been reported that in the shock induced collapse of bubbles in a free field, peaks in pressure are found not only in the downstream propagating water-hammer wave [3]. Locally high pressure regions also develop upstream of the bubble, following the collapse of the bubble remains and the late-time wave interactions. With respect to erosion, these mechanisms are not of importance when the incident wave propagates in a direction that is normal to the surface. However, in the case where the shockwave impacts the bubble from a different direction, the aforementioned mechanisms could be inducing loads of non-negligible magnitude on the nearby surface.

\section{Results}

A front tracking method is employed for the localization of the interface [4]. The flow is modeled by the compressible Euler equations. A fifth-order WENO scheme is used for the convective terms, while integration is carried out by a second-order Runge-Kutta method. The fluids are modeled by the stiffened polytropic equation of state, which reduces to the ideal gas law in the case of air.

A cylindrical air bubble of diameter $\mathrm{d}=0.001 \mathrm{~m}$ is placed in a volume of water next to a solid surface. The distance of the bubble center from the wall is given in terms of the non-dimensional parameter $\gamma=\delta / R$, where $\delta$ is the distance from the wall and $R$ the bubble radius. The computational domain has a size of $x \in[0 \mathrm{~m}, 0.002 \mathrm{~m}], y \in[0 \mathrm{~m}, 0.0025 \mathrm{~m}]$ and is discretized with a resolution that yields a number of 200 points per initial bubble radius. The bubble center is placed along the $y=0.00075 \mathrm{~m}$ line. Both fluids are initially at rest at atmospheric pressure. A shockwave of strength $\mathrm{p}=1 \mathrm{GPa}(\mathrm{M} \approx 1.42)$ propagates from the lower boundary $(\mathrm{y}=0 \mathrm{~m})$ of the domain. The wall at $\mathrm{x}=0 \mathrm{~m}$ is modelled by appropriate reflecting conditions, while non-reflecting conditions are set on the remaining boundaries.

We consider the case where $\gamma=1.25$. As the incident shock impacts the bubble interface a rarefaction wave is reflected back in the liquid. This wave gets reflected at the wall and expands the liquid to the point that it starts to cavitate. The density gradient fluctuations in the vicinity of the solid surface are an artifact of the employed pressure cutoff model, which in general has little to no effect on the overall flow dynamics. The delayed compression of the wall-adjacent bubble half can be observed at the moment just before the impact of the main jet (figures 1(a), 2(a)). In the same frame, we may observe how the shockwave in the vicinity of the wall runs slower and is weaker than its free-field counterpart, following its interaction with the reflected rarefaction wave. In the following frame (figures 1(b), 2(b)), we observe the water-hammer shockwave and the delayed collapse of the lobe that is close to the wall. The microjetting

*Corresponding Author, Nikolaos Bempedelis: nikolaos.bempedelis.16@ucl.ac.uk 


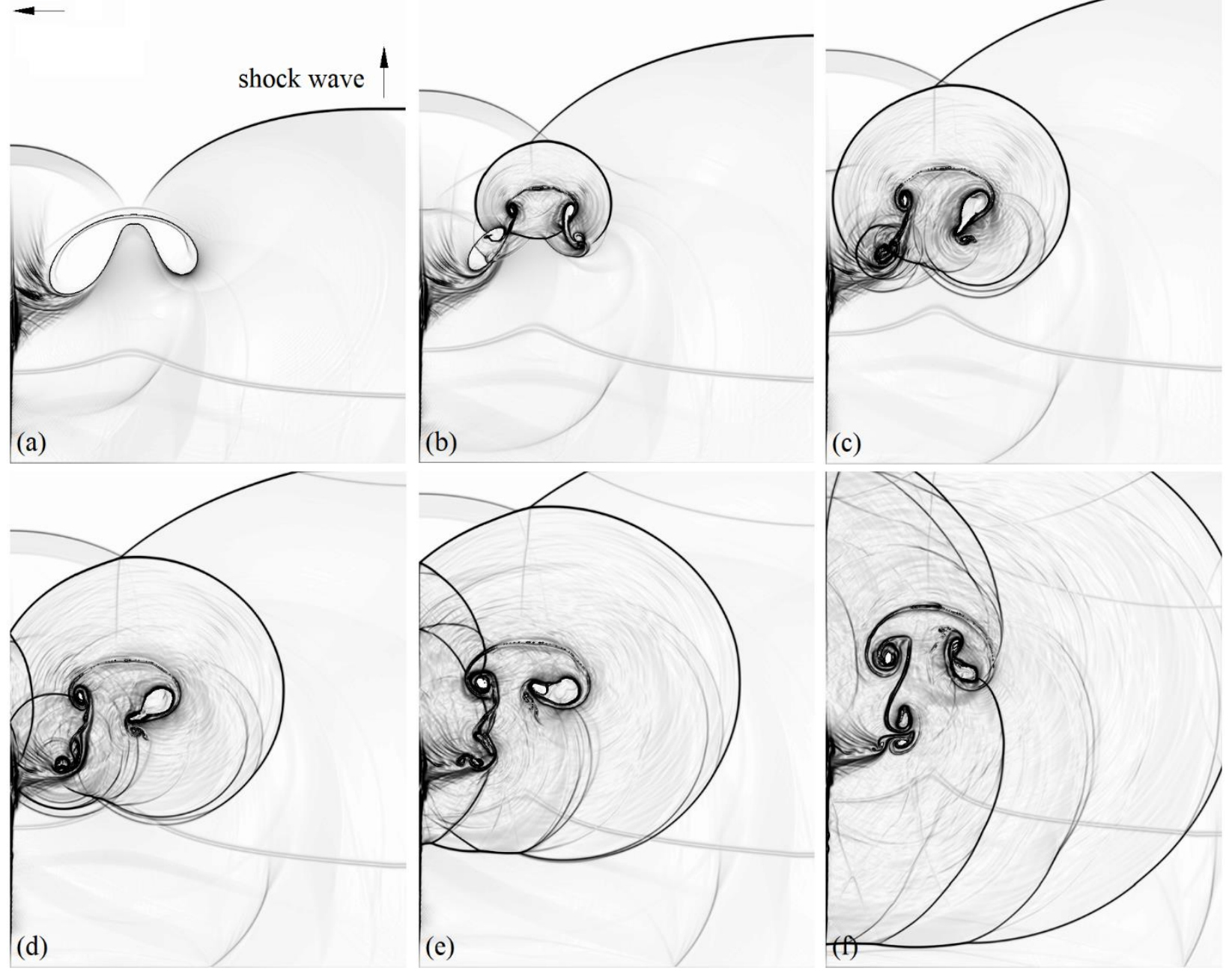

Figure 1:Magnitude of density gradient contours at various time instants after shock impact, $\gamma=1.25$.

(a) $\mathrm{t}=660 \mathrm{~ns}$, (b) $\mathrm{t}=820 \mathrm{~ns}$, (c) $\mathrm{t}=940 \mathrm{~ns}$, (d) $\mathrm{t}=1010 \mathrm{~ns}$, (e) $\mathrm{t}=1120 \mathrm{~ns}$, (f) $\mathrm{t}=1340 \mathrm{~ns}$.

that is formed following the impact of the main jet at the bubble walls is observed on the wall-adjacent lobe only, as it has already impacted the sides of the wall-distal lobe, which is subsequently getting collapsed by the water-hammer shockwave. In the third frame (figures 1(c), 2(c)), the flow field is depicted right before the water-hammer wave impacts the wall. The waves that were formed upon the collapse of the wall-adjacent lobe follow shortly after, and are shown to interact with the reflected water-hammer shock and the wall at the fourth frame (figures 1(d), 2(d)). The gas in the wall-distal lobe has started expanding, whereas its motion is strongly vortical. The region of cavitating liquid is reduced as the water-hammer shockwave traverses it and raises the pressure. The bubble remains get compressed once again by the reflected shocks. In the final frame (figures 1(f), 2(f)), we observe the waves running along the solid surface while decaying in strength. Strong interactions and reflections have ceased. Due to the multiple interactions and shock passages, the gas in the wall-adjacent lobe does not expand as its right counterpart.

The interface of the bubble is depicted in figure 3 at four different instants after shock impact (starting from the moment of jet impact on the bubble wall and following increments of 100ns). In these figures, results from simulations with different stand-off parameters have been superposed on those from a bubble collapsing in a free field. We observe that the dynamics of the first phase (i.e. until the jet impact) are not severely altered. Even though the wall-adjacent lobe is compressed with delay, there is no significant effect on the time that the jet takes to impact the far bubble wall. However, in later stages, the delay gets pronounced for the dynamics of both lobes. As the stand-off parameter decreases, the generated vorticity is reduced. In the case where $\gamma=1.02$ the wall-adjacent upper half of the bubble is practically stagnant. As a result, cavitation is observed only upstream of the bubble. In general, the patterns of the collapse are similar for all stand-off parameters; they just manifest in different intensity and dynamics. 

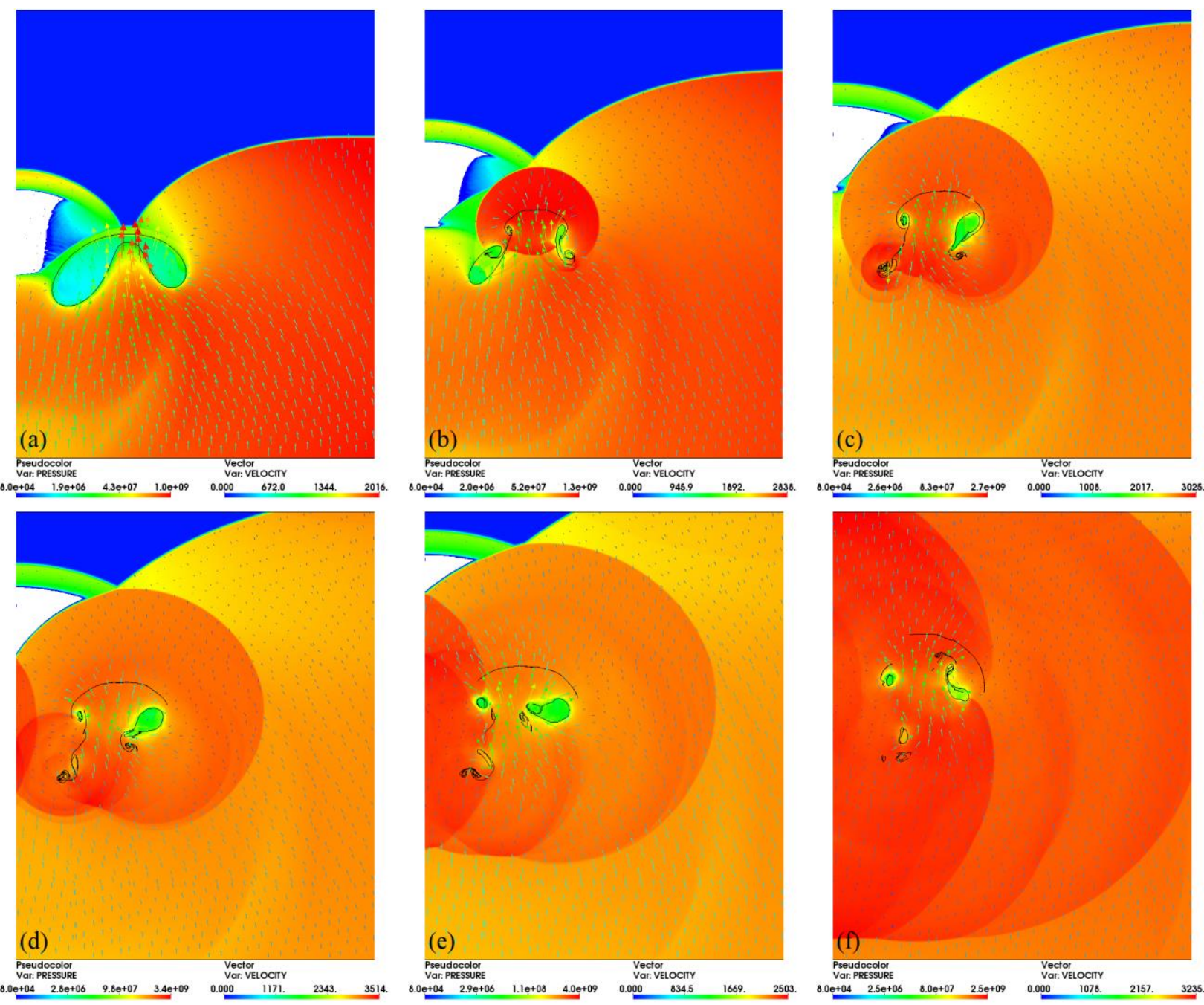

Figure 2: Pressure contours and velocity vectors at various time instants after shock impact, $\gamma=1.25$. Regions of cavitation are shown in white (a) $t=660 n s$, (b) $t=820 n s$, (c) $t=940 n s$, (d) $t=1010 n s$, (e) $t=1120 n s$, (f) $t=1340 n s$.

With respect to erosion, the pressure induced on the wall along time is presented in figure 4. Although the plotted results are from the $\gamma=1.25$ case, the same mechanisms may be identified in all other simulations. However, their relative importance, magnitude and dynamics vary with the stand-off parameter $\gamma$. A first load is induced on the surface following the impact of the water hammer shockwave (at $\mathrm{t}=960 \mathrm{~ns}$ after shock impact). However, this is not a direct impact as in the normal-to-the-wall collapse, and the resulting pressures are weak. The wall experiences a first significant pressure load when impacted by the waves formed during the collapse of the wall-adjacent bubble half (around $t=1000 \mathrm{~ns}$ after shock impact). The intensity of these waves at impact is increasing as the bubble gets closer to the wall. This is expected as the waves originate closer to the wall and have therefore not significantly decayed in strength. Prior to impacting the wall, these waves might experience an increase in strength, in case of interaction with the reflection of the water-hammer shockwave. The peak in the load around $t=1120 \mathrm{~ns}$ occurs after this type of interaction. At later times, the interactions continue as the waves run along the wall. However, all these late-time interactions and reflections are of lower intensity and the waves propagate away from the wall while decaying in strength.

The maximum wall pressures recorded for all simulated stand-off parameters are presented in figure 4 . The results are compared against the respective normal-to-the-wall collapse configurations. The induced pressures are clearly not negligible in magnitude but are smaller than a direct water-hammer shock impact by a percentage of about $\approx 50-60 \%$. 


\begin{tabular}{|l|}
$-\gamma=1.02$ \\
$-\gamma=1.25$ \\
$-\gamma=1.50$ \\
$-\gamma=1.75$ \\
— Free field
\end{tabular}

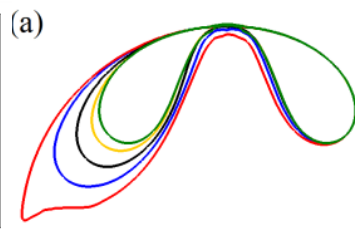

(b)

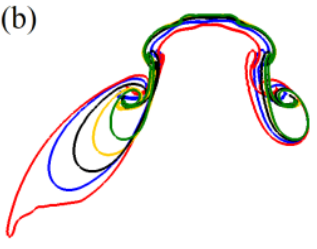

(c)

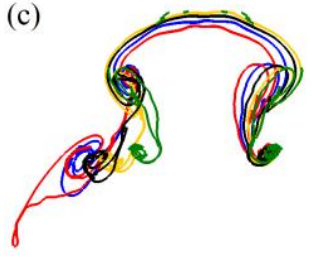

(d)

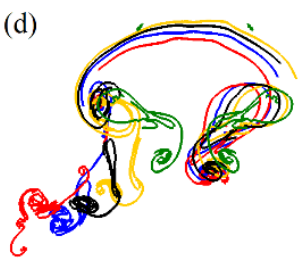

Figure 3: Bubble interface at four different time instants after shock impact for various stand-off parameters. (a) $\mathrm{t}=680 \mathrm{~ns}$, (b) $\mathrm{t}=780 \mathrm{~ns}$, (c) $\mathrm{t}=880 \mathrm{~ns}$, (d) $\mathrm{t}=980 \mathrm{~ns}$.
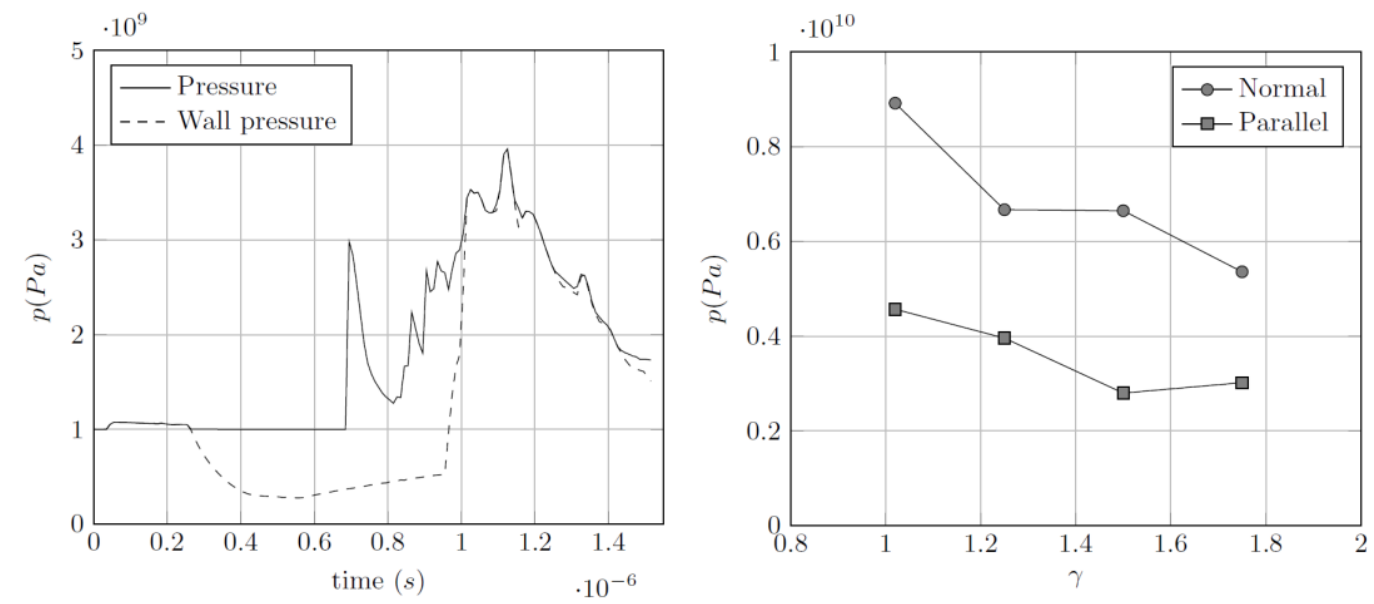

Figure 4: (left) Maximum pressure along time after shock impact, $\gamma=1.25$, (right) maximum wall pressures for various stand-off parameters.

\section{Conclusions}

In the present work we studied the collapse of a gas bubble by a shockwave propagating in a parallel-to-the-wall direction. The shockwave weakens and slows down in the vicinity of the wall due to its interaction with the reflected rarefaction wave, whereas a region of cavitation develops between the solid boundary and the bubble. The walladjacent half of the bubble does not get as compressed as its wall-distal counterpart. The dynamics of the post-jet impact stage are asynchronous; the wall-distal lobe collapses first followed by its wall-adjacent counterpart. Following the reflection of the waves at the solid surface the bubble remnants get collapsed again. With respect to erosion, we demonstrated that the peak wall pressures are generated at the impact of the shocks that are formed during the collapse of the wall-adjacent bubble remains. The loading was shown to be comparable in magnitude to cases of normal-tothe-wall shock induced collapse. In the future, the collapse of cavity arrays will be considered, since pressure is amplified in such configurations. Moreover, the investigation will be extended to the case of spherical bubbles, where the focusing (and hence the pressure) is also more intense.

\section{Acknowledgments}

This project has received funding from the European Union Horizon 2020 Research and Innovation programme, grant agreement No 675676.

\section{References}

[1] Johnsen, E., Colonius, T. (2008). Shock-induced collapse of a gas bubble in shockwave lithotripsy. J. Acoust. Soc. Am, 124(4). [2] Jamaluddin, A.R., Ball, G.J., Turangan, C.K., Leighton, T.G. (2011). The collapse of single bubbles and approximation of the far-field acoustic emissions for cavitation induced by shock wave lithotripsy. J. Fluid Mech, vol. 677, pp. 305-341.

[3] Hawker, N.A., Ventikos, Y. (2012). Interaction of a strong shockwave with a gas bubble in a liquid medium: a numerical study. J. Fluid Mech, vol. 701, pp. 59-97.

[4] Bo, W., Liu, X., Glimm, J., Li, X. (2011). A robust front tracking method: verification and application to simulation of the primary breakup of a liquid jet. SIAM J. Sci. Comput., 33(4). 